\title{
Control Changes the Way We Look at the World
}

\author{
Wen Wen ${ }^{1,2}$ and Patrick Haggard ${ }^{1}$
}

\begin{abstract}
The feeling of control is a fundamental aspect of human experience and accompanies our voluntary actions all the time. However, how the sense of control interacts with wider perception, cognition, and behavior remains poorly understood. This study focused on how controlling an external object influences the allocation of attention. Experiment 1 examined attention to an object that is under a different level of control from the others. Participants searched for a target among multiple distractors on screen. All the distractors were partially under the participant's control (50\% control level), and the search target was either under more or less control than the distractors. The results showed that, against this background of partial control, visual attention was attracted to an object only if it was more controlled than other available objects and not if it was less controlled. Experiment 2 examined attention allocation in contexts of either perfect control or no control over most of the objects. Specifically, the distractors were under either perfect (100\%) control or no (0\%) control, and the search target had one of six levels of control varying from $0 \%$ to $100 \%$. When differences in control between
\end{abstract}

\section{INTRODUCTION}

The sense of control refers to the feeling of controlling our own actions and, through them, external events (Haggard \& Chambon, 2012). Numerous studies focused on the question on how sense of control arises and have revealed important cognitive components including intention, selection between action alternatives, voluntary motor command, and sensory feedback (see Haggard, 2017, for a review). However, the questions on why the sense of control is important and how it influences wider cognitive systems of perception and behavior remain poorly understood. This study investigated how control over an external object influences visual attention. Attention is the process of focusing perception on specific objects through both bottom-up and top-down processes (Schneider \& Shiffrin, 1977). Attention widely influences our perception of the rich and complex external world. On the other hand, the sense of control is an important element for recognizing our connection with the external world. It may, therefore, potentially guide our attention over the external world and consequently influence our perception. Strong effects of control on visual attention are

${ }^{1}$ University College London, ${ }^{2}$ University of Tokyo the distractors and the target were small, visual attention was now more strongly drawn to search targets that were less controlled than distractors, rather than more controlled, suggesting attention to objects over which one might be losing control. Experiment 3 studied the events of losing or gaining control as opposed to the states of having or not having control. ERP measures showed that P300 amplitude proportionally encoded the magnitude of both increases and decreases in degree of control. However, losing control had more marked effects on P170 and P300 than gaining an equivalent degree of control, indicating high priority for efficiently detecting failures of control. Overall, our results suggest that controlled objects preferentially attract attention in uncontrolled environments. However, once control has been registered, the brain becomes highly sensitive to subsequent loss of control. Our findings point toward careful perceptual monitoring of degree of one's own agentic control over external objects. We suggest that control has intrinsic cognitive value because perceptual systems are organized to detect it and, once it has been acquired, to maintain it.

broadly consistent with enactive theories of perception (Goodale, 2014; Goodale \& Milner, 2004; O'Regan, 1992).

The sense of control is closely associated with the sense of agency (i.e., the feeling of being the agent of control). Prior studies have suggested that the sense of agency exhibits a hierarchical relationship with goal-level control and perceptual-motor control (Kumar \& Srinivasan, 2014, 2017). Specifically, to achieve a goal, people may take a series of actions, possibly extending over a long period or an entire lifespan. In health and occupational psychology, the capacity to take such decisions of longterm importance is termed "sense of agency." In addition, at any given moment, they can also perceive proximal effects of actions, which lie spatiotemporally close to actions. The sense of control is based on the recognition of the regularity of proximal action-effect relations. On the other hand, the sense of agency can refer both to the sense of control for each single movement and to control over wider, longer-term outcomes (Wen, Yamashita, \& Asama, 2015a, 2015b, 2017; Metcalfe, Eich, \& Miele, 2013; Metcalfe \& Greene, 2007). In this study, we focus on the sense of control, which is based on the perception on the action-effect regularity.

The processes underlying the sense of control are widely examined with the framework of comparator models. The 
comparator model (Blakemore, Wolpert, \& Frith, 1998, 2002; Wolpert \& Flanagan, 2001; Frith, Blakemore, \& Wolpert, 2000; Wolpert \& Ghahramani, 2000; Blakemore, Frith, \& Wolpert, 1999) suggests that the subjective feeling of control is produced via the comparison between a predicted state basing on the efference copy of motor command and the actual sensory feedback. Therefore, if there is a discrepancy between the actual sensory feedback and the internal predicted state, the comparator produces a mismatch signal or prediction error. The model assumes that this prediction error is the most salient experience during control episodes and corresponds to a feeling of diminished control or lack of agency. This view places error monitoring at the core of sense of control. Several neuroscientific studies produced evidence consistent with this view (e.g., Chambon, Wenke, Fleming, Prinz, \& Haggard, 2013; Kühn, Brass, \& Haggard, 2013; Farrer et al., 2003, 2008; Ogawa \& Inui, 2007; Farrer \& Frith, 2002). However, the effects of detecting such errors on wider cognition remain unclear. Presumably, detecting diminished control acts as a trigger to executive and motor functions that aim to reassert control (Botvinick, Niv, \& Barto, 2009; Botvinick, Cohen, \& Carter, 2004). However, because reduced control is potentially of high importance to the organism, it seems likely that detecting control errors triggers wider cognitive effects, beginning with perceptual processing. A previous EEG study showed that unpredicted outcomes were automatically monitored and elicited a feedback-related negativity even though the outcome was task irrelevant (Band, van Steenbergen, Ridderinkhof, Falkenstein, \& Hommel, 2009), highlighting the processes of automatic control-error monitoring.

In this study, we focus particularly on how control over an external object influences attention. Previous studies suggested a link between attention and the sense of control (Kumar, Manjaly, \& Sunny, 2015; Salomon, Lim, Kannape, Llobera, \& Blanke, 2013). Salomon et al. (2013) reported a phenomenon of "self pop-out" in visual search; stimuli controlled by participants' voluntary actions showed a pop-out effect in the visual search. Kumar et al. (2015) found that a motion onset or color change that was proceed by an action that controlled a specific object facilitated the visual search of that object. These results provided evidences of bottom-up attention drawn to objects that were actively controlled. However, it is unclear whether the monitoring of lack or loss of control also attracts attention.

In summary, although there is evidence that the sense of control might somehow affect our attention allocation (e.g., Kumar et al., 2015; Salomon et al., 2013), the overall picture of the link between monitoring one's own control and perceptual processing remains unclear. In this study, we examined attentional allocation related to both the state of control (i.e., control differs among objects) and also to a change of control (i.e., control over an object decreases or increases). First, we investigated these questions by presenting several visual objects that could
Table 1. Definition of Terms that Describe the Experimental Conditions

\begin{tabular}{|c|c|}
\hline Control difference & $\begin{array}{l}\text { The difference in level of control } \\
\text { between one stimulus and the } \\
\text { majority of stimuli }\end{array}$ \\
\hline Control change & $\begin{array}{l}\text { The difference in level of control over } \\
\text { a focused stimulus between the } \\
\text { beginning and the end of a trial }\end{array}$ \\
\hline Control context & $\begin{array}{l}\text { The level of control over the majority } \\
\text { of the stimuli }\end{array}$ \\
\hline Target & $\begin{array}{l}\text { The visual search target (i.e., the circle } \\
\text { with a single gap) }\end{array}$ \\
\hline Distractor & $\begin{array}{l}\text { The stimulus that does not have the } \\
\text { specific feature of visual search } \\
\text { (i.e., the circle with two gaps) }\end{array}$ \\
\hline Exception & $\begin{array}{l}\text { The only stimulus that is under a } \\
\text { different level of control than the } \\
\text { majority of the stimuli }\end{array}$ \\
\hline
\end{tabular}

be controlled to varying degrees by the participant's movement and by using visual search paradigms to investigate how those objects were perceptually processed in Experiments 1 and 2. Then, we used ERPs to examine the subprocesses of attention allocation triggered when the level of control over a visual object suddenly changed. We hypothesize that an object that is under control is prioritized for perceptual processing when people do not have much control over the external world; once people have acquired a high level of control over the external world, monitoring for lack or loss of control takes high attentional priority.

To make the terminology clear, the terms that we used to describe the experimental conditions are defined in Table 1 .

\section{EXPERIMENT 1}

\section{Methods}

\section{Participants}

Twenty-three healthy volunteers were recruited from a participant database. One participant was excluded because of not following instructions. Another four participants were excluded because of failure in agency judgment $\left(d^{\prime}\right.$ of the signal detection theory was less than 0.1 for distinction between $0 \%$ and $100 \%$ control). Therefore, results from 18 participants (mean age $=22.7$, range $=$ $19-38, S D=4.3$, 14 women) were used for the analysis. The sample size was based on a power calculation $(\alpha=$ .05 , power $(1-\beta$ error $)=.95)$, with an effect size estimated from the results of a pilot experiment $(n=3)$ using G*Power 3 (Faul, Erdfelder, Lang, \& Buchner, 2007). All but one of the participants were right-handed. The left-handed participant reported daily use of the 
computer mouse with the right hand and used the right hand to move the mouse in the task. All participants reported normal or corrected-to-normal visual acuity. The study was approved by the local ethics committee (University College London). All participants provided written informed consent before participation and received $\$ 7.5$ per hour as reimbursement.

\section{Task}

Figure 1 shows a timeline of a trial in the experimental task. ${ }^{1}$ At the start of each trial, four or six $18-\mathrm{mm}$ circles with 2.5-mm black border appeared at random positions on a $338 \times 270 \mathrm{~mm}$ (width $\times$ height) gray background. Once participants started to move the mouse, all the circles started to move. The velocity, onset, and offset of the motion of all the stimuli corresponded to participants' mouse movement, but the relation between the mouse movement and the circles' spatial trajectories could vary (see later). In other words, all the circles stayed static if participants did not move the mouse, and all started to move at the same velocity (but in different directions) once participants started to move the mouse. When and only when the circles were moving did their shapes change. For most circles, 2.5-mm gaps appeared on both sides of the circles when they moved and disappeared when they were static. However, one circle had a gap at the left side only. Participants searched for the single-gap target circle among the double-gap distractors while continuously moving the mouse to make the gap visible.

The movements of the distractors were under 50\% control, meaning that the participant's mouse movement was mixed with prerecorded movements of other participants on a 50/50 basis. The other participants' movements were randomly selected sections from 10,000 prerecorded continuous mouse movements in similar tasks. Temporal information of prerecorded movements was discarded, and only spatial information was used. Specifically, at each moment when participants moved the mouse, the movement of the stimuli was generated from a combination of the online mouse movement and prerecorded movement with a specific ratio according to the levels of control. Different sections of movement were applied randomly to different distractors. Therefore, all the stimuli moved in different directions. The search target was under $0 \%, 40 \%, 60 \%$, or $100 \%$ control, where $100 \%$ control means an exact correspondence between mouse movement and visual target movement. To measure the individual difference in visual search, we also included two baseline conditions (with set size 4 and 6 , respectively) in which all the stimuli including the search target were under 50\% control.

Participants were instructed to find the single-gapped search target as quickly and accurately as possible. They used the spacebar on a keyboard to indicate that they found the search target, at which point all circles became static and closed. They then pressed a number key to identify the target. Finally, they used three designated keys to report whether they felt more or less control or the same degree of control over the search target as the other circles. If the target was not detected within $5 \mathrm{sec}$ of the first mouse movement, the trial was terminated.

There were four within-participant independent variables. The first one was the "extent" of control difference (small or large) between the search target and the distractors. The control over the distractors was always 50\%; therefore, the difference between target and distractor control was large when the search target was under $0 \%$ or $100 \%$ control and was small when the search target was under $40 \%$ or $60 \%$ control. The second variable was the "direction" of control difference: Search targets with $0 \%$ or $40 \%$ were less controlled than the distractors, whereas search targets of $60 \%$ or $100 \%$ were under more control. The third independent variable related to the timing of the change in control. In half the trials, the differential control over target and distractors was present throughout the trial, whereas in the other half of the trials, the differential control over the target and the appearance of the gaps were both introduced at a random latency between 1.5 and $2 \mathrm{sec}$. This factor allowed us to distinguish between effects of control as
Figure 1. Timeline of a trial in Experiment 1.

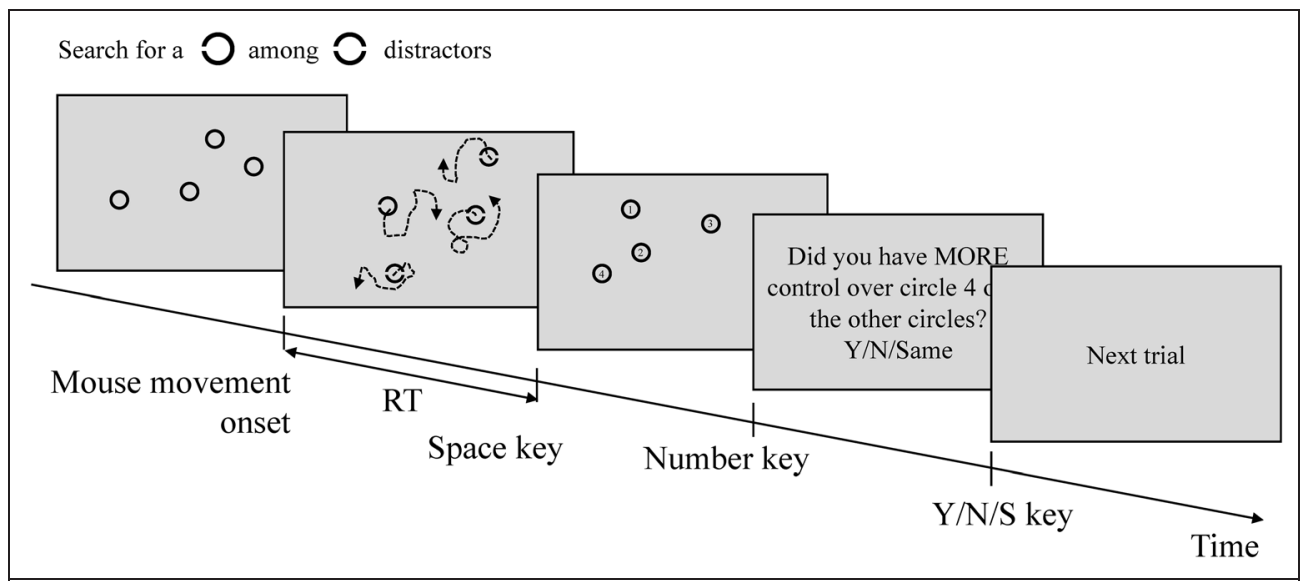


Table 2. The Actual Level of Control over the Target in Each Control Condition of Experiment 1

\begin{tabular}{|c|c|c|c|}
\hline \multicolumn{4}{|c|}{ Fixed Level of Control over Target } \\
\hline \multicolumn{2}{|c|}{$\begin{array}{c}\text { Small Difference between the } \\
\text { Target and Distractors }\end{array}$} & \multicolumn{2}{|c|}{$\begin{array}{c}\text { Large Difference between the } \\
\text { Target and Distractors }\end{array}$} \\
\hline $\begin{array}{l}\text { Target }< \\
\text { Distractor }\end{array}$ & $\begin{array}{c}\text { Target }> \\
\text { Distractor }\end{array}$ & $\begin{array}{l}\text { Target }< \\
\text { Distractor }\end{array}$ & $\begin{array}{l}\text { Target }> \\
\text { Distractor }\end{array}$ \\
\hline $40 \%$ & $60 \%$ & $0 \%$ & $100 \%$ \\
\hline \multicolumn{4}{|c|}{ Changing Level of Control over Target } \\
\hline \multicolumn{2}{|c|}{$\begin{array}{c}\text { Small Change over } \\
\text { the Target }\end{array}$} & \multicolumn{2}{|c|}{$\begin{array}{c}\text { Large Change over } \\
\text { the Target }\end{array}$} \\
\hline Decrease & Increase & Decrease & Increase \\
\hline $50 \% \rightarrow 40 \%$ & $50 \% \rightarrow 60 \%$ & $50 \% \rightarrow 0 \%$ & $50 \% \rightarrow 100 \%$ \\
\hline
\end{tabular}

a state versus as an event. The fourth variable was the set size ( 4 or 6 ). We manipulated the number of stimuli to test whether the detection of control is automatic and parallel, as reported in Salomon et al. (2013). To sum up, Table 2 shows the actual level of control over the target in each condition (set size is omitted in Table 2).

\section{Procedure}

Participants were tested individually in a quiet testing room, seated on a chair positioned approximately $50 \mathrm{~cm}$ from a 17-in. LCD monitor (resolution $1280 \times 1024$ pixels at $75 \mathrm{~Hz}$ ). Having received an explanation regarding the requirements of the experimental task, participants practiced for 36 trials, containing two trials of each condition. During the practice, the experimenter supervised the method of mouse movement, suggesting smooth and continuous mouse movement to make the gaps on the stimuli continuously visible. The actual task contained 360 trials, including 20 repeats of each condition (2 [extent of difference] $\times 2$ [direction of difference] $\times 2$ [changing in control] +1 [baseline] $) \times 2$ (set size), and were divided into four blocks, each containing 90 trials. The order of trials was randomized. Participants took short breaks between blocks according to their requests. The experiment lasted for $60 \mathrm{~min}$ on average.

\section{Results}

The average accuracy in the visual search task was $95.5 \%$ $(S D=3.0 \%$, the proportion of trials with the incorrect specification of target was 3.6\%, and the proportion of time-out trials was $0.9 \%$ ), indicating that the participants gave accuracy high priority and speed-accuracy trade-off did not occur. Results from the trials with the target correctly located were used for analyses. First, to examine the overall effect of set size, we applied a 2 (Extent of control difference, small or large) $\times 2$ (Direction of control difference, less or more) $\times 2$ (Change in control, consistent or changed) $\times 2$ (Set size of 4 or 6 ) repeatedmeasures ANOVA on the RT from all the conditions, except the two baseline. The main effect of Set size was significant, $F(1,17)=108.86, p<.001$, partial $\eta^{2}=.87$, but it did not interact with any other factors. The results thus indicated that the visual search was serial, so control over the target did not appear to "pop-out." Because Set size did not interact with the other factors, we averaged results from trials with different set size and removed this factor from further analyses. In addition, the main effect of Direction of control difference, $F(1,17)=34.90, p<.001$, partial $\eta^{2}=.67$, the interaction between Extent and Direction of difference, $F(1,17)=26.23, p<.001$, partial $\eta^{2}=$ .61 , and the interaction between Change in control and Direction of control difference, $F(1,17)=4.56, p=$ .048 , partial $\eta^{2}=.21$, were significant.

Figure 2 shows the average RT for each condition. For the analysis, we used the difference in RT between the experimental conditions and the baseline condition as the "effect of control," allowing us to exclude the influence of individual differences in visual search. A 2 (Extent of control difference, small or large) $\times 2$ (Direction of control difference, less or more) $\times 2$ (Change in control, consistent or changed) repeated-measures ANOVA revealed a significant main effect of Direction of control difference, $F(1,17)=35.93, p<.001$, partial $\eta^{2}=.68$, a significant interaction between Direction and Extent of control difference, $F(1,17)=25.31, p<.001$, partial $\eta^{2}=.60$, and a significant interaction between Direction of control difference and Change in control, $F(1,17)=$ $5.06, p=.038$, partial $\eta^{2}=.23$. Visual search was faster when the control over the target was greater than control over distractors, compared with vice versa. Post hoc comparisons (Bonferroni-adjusted tests) on the interaction between the direction and extent of control difference showed that large differences in control facilitated visual search more than small differences in the condition when the control over the target was more than the distractors (100\% vs. $60 \%$ control, $p<.001$ ), but not when the control over the target was less than the distractors ( $0 \%$ vs. $40 \%$ control, $p=.170$ ). In other words, increased control over the target attracted more attention, but diminished control did not. Finally, the interaction between direction of control difference and change of control indicated that an unpredictable change in control facilitated visual search when the control decreased, compared with when control increased. This suggests that, although high levels of control generally facilitate attention, loss of control may also be an important event for attentional processing. However, post hoc comparisons did not find any significant differences between conditions. In addition, the main effect of Extent of control difference, $F(1,17)=1.11, p>.250$, partial $\eta^{2}=.06$, the main effect of Change in control, $F(1,17)=0.26, p>.250$, 


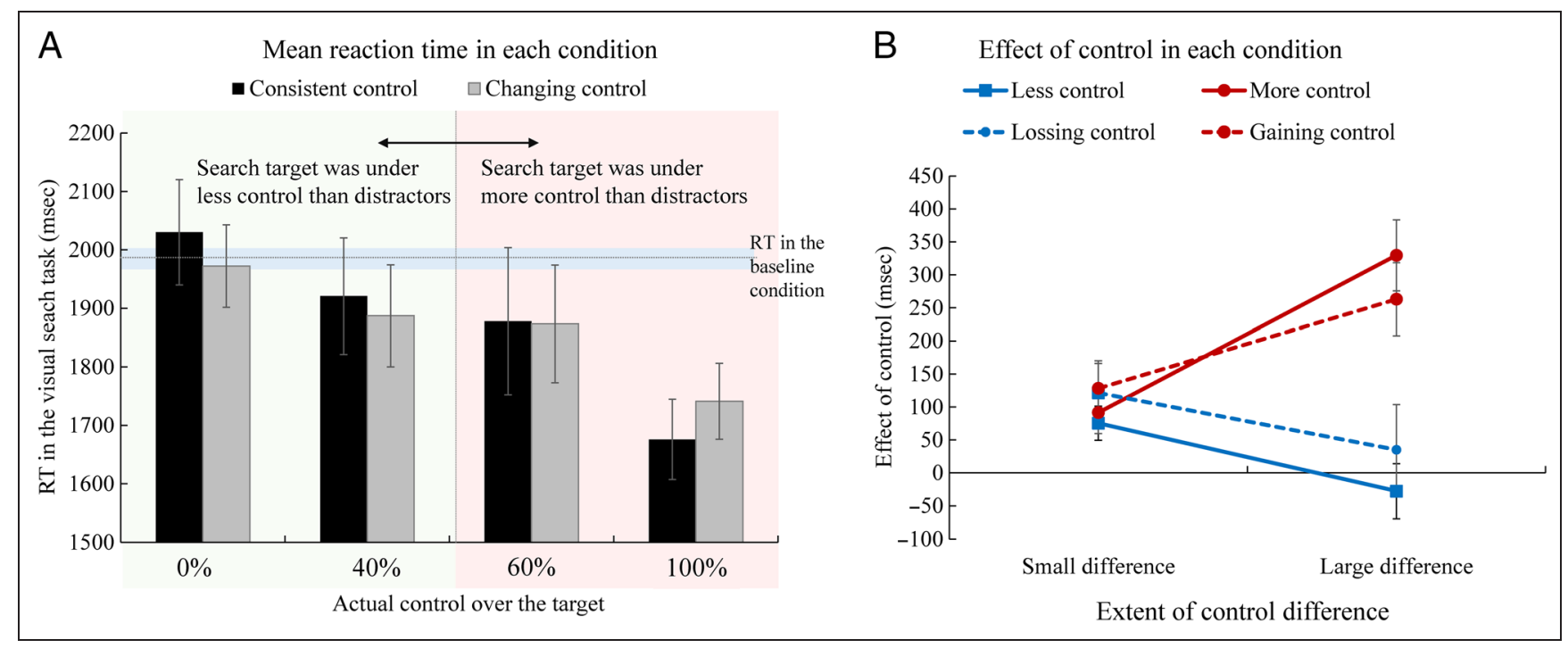

Figure 2. Mean RT (A) and the effect of control (B) in the visual search task of Experiment 1. Error bars and blue square represent standard errors.

partial $\eta^{2}=.02$, the interaction between Extent of control difference and Change in control, $F(1,17)=0.96, p>.250$, partial $\eta^{2}=.05$, and the three-way interaction, $F(1,17)=$ $2.66, p=.121$, partial $\eta^{2}=.14$, were nonsignificant.

Figure 3 shows participants' subjective judgments regarding their degree of control over the target for each condition and the values of $d^{\prime}\left(Z_{\text {hit }}-Z_{\text {false-alarm }}\right)$. We conducted a 2 (Extent of control difference, small or large) $\times 2$ (Direction of control difference, less or more) $\times$ 2 (Change in control, consistent or changed) repeatedmeasures ANOVA on $d^{\prime}$ values. The main effect of Extent of control difference was significant, indicating that the judgment in control was more accurate when the actual control over the target was more different from the distractors, $F(1,17)=25.42, p<.001$, partial $\eta^{2}=.60$. The main effect of Direction of control difference was nonsignificant, indicating that people were equally good at detecting more and less control, $F(1,17)=2.26, p=$ .151 , partial $\eta^{2}=.12$. The main effect of Change in control was nonsignificant, $F(1,17)=0.28, p>.250$, partial $\eta^{2}=$ .02 , suggesting that judgments of control were unaffected by whether level of control changed during the trial or not. In addition, the interaction between the extent and direction of control difference, $F(1,17)=21.27, p<$ .001 , partial $\eta^{2}=.56$, and the interaction between change in control and direction of control difference, $F(1,17)=$ $18.59, p<.001$, partial $\eta^{2}=.52$, were significant. Post hoc analyses (Bonferroni-adjusted tests) on the interaction between the extent and direction of control difference showed that, when the difference in control was large, the perception of control over the target was more sensitive when it was under more control $(p=.001)$. When

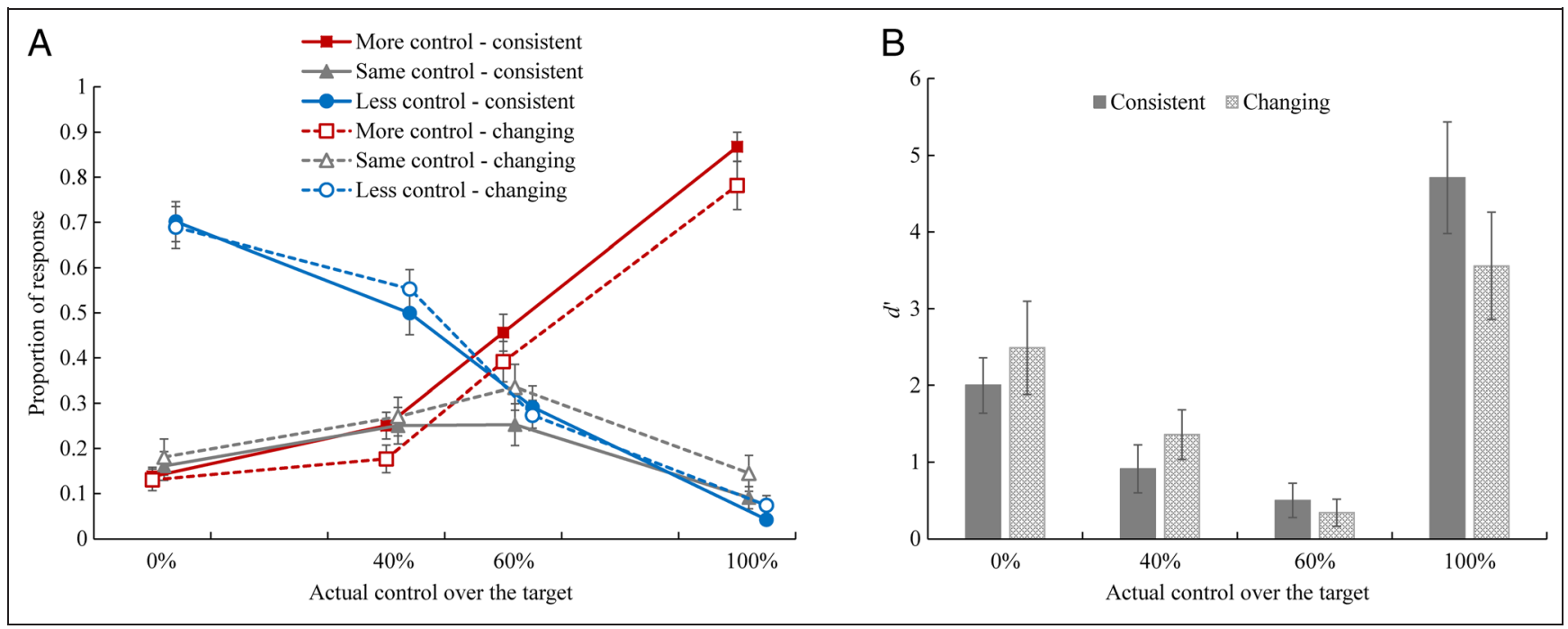

Figure 3. Control judgment in Experiment 1. Error bars represent standard errors. (A) Proportion of each control response (i.e., more, same, or less) in Experiment 1. (B) $d^{\prime}$ in each control condition. 
the difference in control was small, the perception of control over the target did not differ between the conditions of more and less control $(p>.250)$. Post hoc comparisons (Bonferroni-adjusted tests) on the interactions between change in control and direction in control difference revealed better perceptual sensitivity for more control (i.e., 60\% and 100\%) than less control (i.e., 0\% and $40 \%$ ) when control over the target was consistent $(p<.001)$, and equal perceptual sensitivity for more and less control when control changed $(p>.250)$. In short, the most important finding from the results of control judgment was that people were equally good at perceiving control regardless of whether the target was under less or more control than the distractors. In addition, a change in control appeared to improve the perceptual sensitivity of control, particularly when the change was a decrease.

Furthermore, hierarchical linear modeling (HLM7; Raudenbush, Bryk, Cheong, Congdon, \& Du Toit, 2011) was used to examine whether the subjective feeling of control was linked to attention allocation and whether this link was influenced by individual differences in sense of control. We used a two-level model: A within-participant level estimated the linear relation across different levels of actual control between the subjective feeling of control (i.e., hit rate of the control judgment, HIT) and the performance in visual search (i.e., the difference in the RT from the baseline condition, RT; Level 1 model: $\mathrm{RT}_{i j}=\beta_{0 j}+\beta_{1 j}$ * $\left(\mathrm{HIT}_{i j}\right)+r_{i j}$, HIT has been centered around the group mean), and a between-participant level estimated the influence of individual differences in sense of control (i.e., the overall average hit rate of control judgment, MHIT) on the slope of the above regression (Level 2 model: $\beta_{0 j}=$ $\left.\gamma_{00}+u_{0 j} ; \beta_{1 j}=\gamma_{10}+\gamma_{11} *\left(\mathrm{MHIT}_{j}\right)\right)$. First, the model revealed a significant correlation between the subjective feeling of control and the performance in visual search (for $\gamma_{10}, p=.003$; Figure 4A). Furthermore, individual differences in judgments of control significantly influenced this correlation (for $\gamma_{11}, p<.001$ ). Participants who were better at detecting control (i.e., higher accuracy in control judgments) benefited from the sense of control to a greater extent in the visual search task, comparing with those who were worse at detecting control. On the other hand, the HLM analysis of the results from the condition in which the target was under less control than the distractors did not reveal any significant correlation or any influences of individual differences on the correlation (for $\gamma_{10}, p>.250$; for $\gamma_{11}, p>.250$; Figure 4B), indicating that the subjective feeling of lack/loss of control did not facilitate visual search.

\section{Discussion}

Experiment 1 investigated whether having control over an object alters perceptual processing of that object both when the level of control over it differed from the other objects and also when the level of control over it suddenly changed to a state that differed from the others. Participants initially always had partial (50\%) control over all the objects in the display, so we could investigate the effects on visual search of more/increased or less/ decreased control of a target object, relative to distractors. When the target was under more control than the distractors, visual search was facilitated by the degree of control over the target. Importantly, the extent of facilitation was correlated with the subjective feeling of control. This provides an interesting and novel validation that subjective feelings of control may track genuine changes in neurocognitive processing and are not merely inferences or illusions (Wegner, 2002). Here, the facilitation of attention by control provides independent evidence of the neurocognitive processes accompanying feelings of control. In addition, the interaction between the direction of target-distractor difference in control and the presence of an event of changing control also affected attentional processing. In particular, the event of suddenly losing a
Figure 4. Individual correlations between the performance in visual search and the subjective feeling of control. Different colors represent individuals (one dot per control level per person, six dots per person). Black lines represent individual correlations. The sense of control was significantly correlated with the performance in visual search when the target was under more control than the distractors (A) but was not when the target was under less control than the distractors (B).

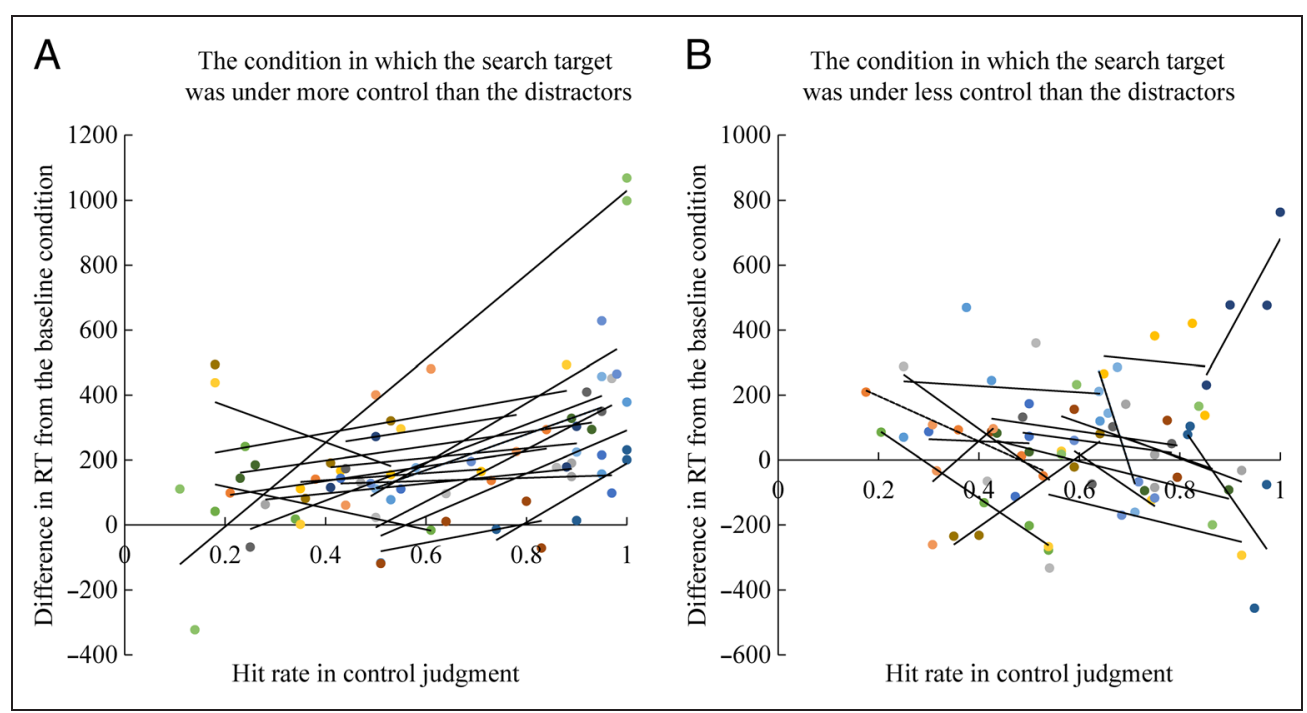


degree of control appeared to have greater effects on attentional processing than suddenly gaining an equivalent amount of control. We further examine this issue in Experiment 3.

The results of the HLM analysis showed that participants who were best at detecting control showed greatest control-related facilitation of visual search. A similar correlation was observed within individuals across trials. We assume that spatial congruency computations that underlie feelings of control in this task play a continuous role in directing attention. However, in contrast to the previous study by Salomon et al. (2013), we failed to find a popout effect of self-controlled object in visual search. Our results showed the detection of control based on spatial congruency is probably serial and requires attentional monitoring, rather than parallel and automatic.

Moreover, once control has been detected and established, a specific cognitive process seems to ensure continuous monitoring of control, with particularly careful attention to situations of loss of control. Experiment 2 aims to investigate this process in more detail. In particular, the level of control over most dots in Experiment 1 was only intermediate (i.e., 50\%). We hypothesized that the process of monitoring for lack or loss of control might be engaged more effectively at higher levels of control. Experiment 2 therefore induced conditions in which the overall control was either perfect (i.e., 100\% control), compared with completely absent (i.e., $0 \%)$. Because information relevant to the self is prioritized in attention (Salomon et al., 2013; Salomon, Szpiro-Grinberg, \& Lamy, 2011; Tacikowski \& Nowicka, 2010; Whiteley, Spence, \& Haggard, 2008; Keenan et al., 1999; Rogers, Kuiper, \& Kirker, 1977), we predict that the experience of having control would be precisely represented, so that a very slight loss of control would strongly attract attention.

\section{EXPERIMENT 2}

\section{Methods}

\section{Participants}

Twenty-two healthy volunteers were recruited from the same participant database as in Experiment 1. One par- ticipant failed to respond within the available time in over half of the baseline trials, resulting in insufficient valid trials. Another participant misunderstood the instructions about how to respond (clicking the mouse rather than pressing the space key). These participants were excluded from the analysis, resulting in a sample size of 20 (mean age $=23.1$, range $=18-40, S D=6.2,15$ women $)$. The sample size was based on a power calculation with an effect size estimated from the results of Experiment 1 (Bonferroni-adjusted $\alpha=.0083$, power $(1-\beta$ error $)=$ .95) using $G^{*}$ Power 3 (Faul et al., 2007). All the participants reported normal or corrected-to-normal visual acuity. One participant was left-handed but reported using the mouse with the right hand daily. The study was approved by the local ethics committee (University College London). All participants provided written informed consent before participation and received $\$ 7.5$ per hour as reimbursement.

\section{Task}

We used a similar visual search task to Experiment 1 (Figure 5). ${ }^{2}$ The stimuli were $10-\mathrm{mm}$ circles with $2-\mathrm{mm}$ border (i.e., smaller than those in Experiment 1), and the set size was 10 . Experiment 2 was designed to directly compare a no-control context and a perfect control context, in which several objects moved together in congruent with the finger movement. To produce comparable task difficulty for the no-control context, uncontrollable objects were also designed to move together in the same direction (different from Experiment 1). Because the visual search should be easier with such grouped motion, we increased the set size to adjust the task difficulty for Experiment 2. In addition, Experiment 2 omitted the factor of change in control to keep the task at a rational length and postponed the issue of examining increases and decreases in control to Experiment 3.

In each trial, most of the circles moved under either $0 \%$ or $100 \%$ control. All stimuli moved together in the same direction except for one exception stimulus, which could be either the target or a distractor for the visual attention task. Therefore, in half of trials the search target was not the exception. This prevented the participant
Figure 5. Timeline of a trial in Experiment 2. See text for explanation.

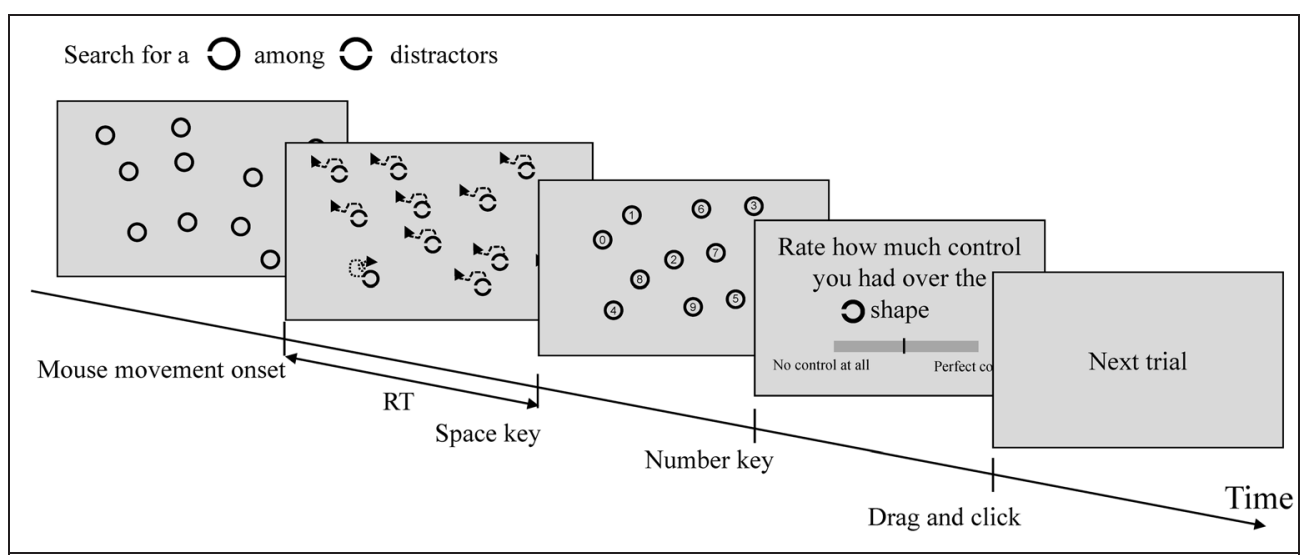


from using a strategy of always attending to the one stimulus that moved differently from the others. The exception stimulus was under 0\%, 20\%, 40\%, 60\%, 80\%, or $100 \%$ control. Participants responded with the space key as soon as possible once they noticed the search target (i.e., the circle with one gap at the left side) and pressed a number key to indicate its location thereafter. Trials were terminated $5 \mathrm{sec}$ after the first mouse movement if the target was not detected. Then they used a mouse to drag a cursor on an on-screen scale ("no control at all" on the left and "perfect control" on the right, in 1-point increments) to rate the extent of control they felt over the search target.

We used a 2 (Control context, 0\% or 100\%) $\times 6$ (Control over the exception, $0 \%, 20 \%, 40 \%, 60 \%, 80 \%$, or $100 \%) \times 2$ (Type of exception, target or distractor) withinindividual design. Our main research question was to identify how the difference in control between the context (i.e., most of the stimuli) and the exception would influence attention in the two different control contexts.

\section{Procedure}

The procedure was similar as that in Experiment 1. Participants performed 48 practice trials, containing two trials of each condition, and 360 trials for the actual task, containing 15 repeats of each condition. The trials were divided into four blocks, each containing 90 trials. The control context ( $0 \%$ or $100 \%)$ was blocked, and the order of control context was counterbalanced between participants. The trial order in each block was randomized. Participants took short breaks between blocks, and the experiment lasted for $60 \mathrm{~min}$ on average.

\section{Results}

The average accuracy in the visual search task was $95.7 \%$ $(S D=3.2 \%$, the proportion of trials with the incorrect identification of target was $1.5 \%$, and the proportion of time-out trials was 2.8\%). The average RT for each condition from the trials of successful visual search (i.e., target was correctly located) is shown in Figure 6. To compare the performance of visual search between the two control contexts, we used control difference (i.e., the difference in level of control between the context and exception) as the $x$ axis. A 2 (Control context, no control or perfect control) $\times 6$ (Control difference, $0 \%, 20 \%$, $40 \%, 60 \%, 80 \%$, or $100 \%$ ) repeated-measures ANOVA on the average RT in the condition when the exception was the search target revealed a significant main effect of Control difference, $F(5,95)=51.29, p<.001$, partial $\eta^{2}=$ .73 , and a significant interaction between Control difference and Control context, $F(5,95)=9.47, p<.001$, partial $\eta^{2}=.33$. The larger the difference in control was, the faster the visual search was. This was not surprising because when the difference in control was large, the search target was more salient. The interaction was more

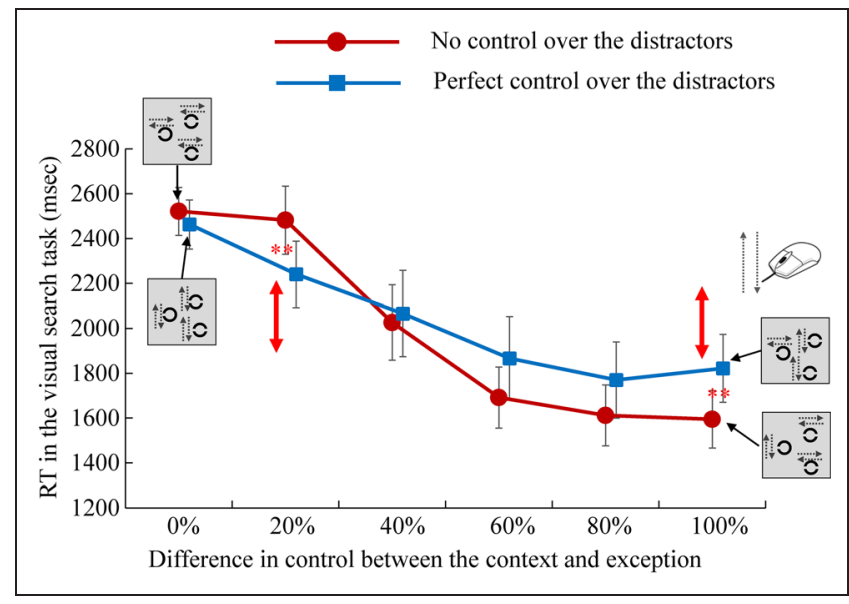

Figure 6. Mean RT in the visual search task of Experiment 2. Error bars represent standard errors. Black squares show the conceptual examples on how the search target and distractors moved like when the mouse moved vertically.

important. Figure 6 shows the nature of this interaction clearly: When participants had perfect control over the majority of objects, attention to the exception object depended only modestly and linearly on the discrepancy in control over it. In contrast, when participants had no control over the majority of objects, the degree of control over the exception object had strong and nonlinear effects on attentional processing. Thus, highly efficient attentional processing was found for exception objects under strong control in the context of no overall control. However, attention was not drawn to an exception object under modest control in the context of no overall control. Put another way, the effects of control on attention were highly asymmetric. When participants had control of the dots, an exception object with very modestly lower level of control successfully captured attention. When participants had no control of the objects, an exception object with having a comparably greater level of control failed to capture attention. Post hoc comparisons using Holm-Bonferroni correction on the difference between control contexts at each level showed that the visual search was faster when the search target was under $20 \%$ less control than distractors at $100 \%$ control, compared with the case when the search target was under $20 \%$ more control than distractors under $0 \%$ control, $t(19)=3.87, p=.001$, Cohen's $d=0.36$. The results supported our hypothesis that people are disproportionately sensitive to small changes of control when they already control an object perfectly. Equally, visual search was slower when the search target was under $0 \%$ control while the distractors were under 100\% control, compared with the case when the search target was under $100 \%$ while the distractors were under $0 \%$ control, $t(19)=$ $-3.16, p=.005$, Cohen's $d=0.36$. This result indicated that full control may have a special status in attentional processes and that this special status may be tuned to detecting decreases in control. The differences between these 


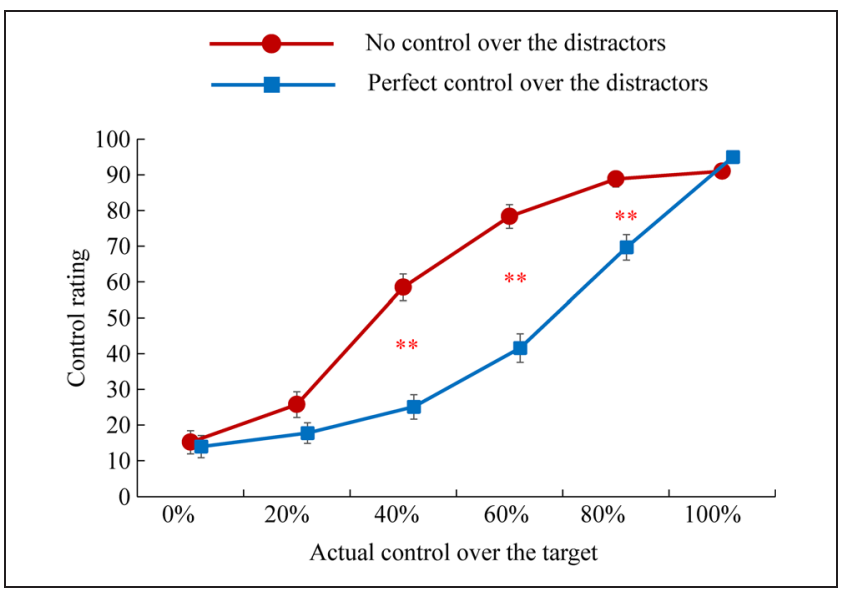

Figure 7. Mean control rating on the target in Experiment 2. The participants felt lower control over the search target when they had perfect control over the distractors than that when they had no control over the distractors.

two asymmetrically opposed control situations were nonsignificant when the difference in control was $0 \%, 40 \%$, $60 \%$, and $80 \%(t(19)=1.23,-0.56,-1.98$, and $-2.44, p=$ $.23, .58, .06$, and .03 , Cohen's $d=0.12,0.05,0.24$, and 0.23 , respectively). The main effect of Control context was not significant, $F(1,19)=1.25, p>.250$, partial $\eta^{2}=$ .06. In addition, the condition in which the exception was a distractor was designed to reduce the using of strategy of always searching for a stimulus moving different from the others. Therefore, the results from these trials were not our main interest.

The average control rating for the search target from the condition in which the exception was the target is shown in Figure 7 . Notice here the $x$ axis represents the actual control over the target (cf. Figure 6 where the $x$ axis represented a difference between the visual search target and the control context). A 2 (Control context, no control or perfect control) $\times 6$ (Actual control, $0 \%, 20 \%, 40 \%, 60 \%, 80 \%$, or $100 \%)$ repeated-measures
ANOVA revealed a significant main effect of Control context, $F(1,19)=18.74, p<.001$, partial $\eta^{2}=.50$, a significant main effect of Actual control, $F(5,95)=303.02, p<$ .001 , partial $\eta^{2}=.94$, and a significant interaction between Control context and Actual control, $F(1,19)=$ 23.55, $p<.001$, partial $\eta^{2}=.55$. Post hoc comparisons using Holm-Bonferroni correction on the interaction revealed that the subjective feeling of control over the search target was significantly lower in the 40\%, 60\%, and $80 \%$ control conditions when the distractors were under perfect control than that when the distractors were under no control $(t(19)=5.39,5.96$, and 4.42, $p$ s $<.001$, Cohen's $d=2.09,2.23$, and 1.45 , respectively). The results showed that people underestimated their degree of control over the target when they already had acquired high levels of overall control over the distractors.

We used structural equation modeling (SEM) to examine the link between the subjective feeling of control and attention allocation. In Experiment 2, because the majority of the stimuli moved together, attention could be attracted by both the salience of the target and the subjective feeling of control over the target. SEM is more appropriate for such multiple variable structures than the HLM used in Experiment 1. The SEM model contained one independent variable-actual control over the target -and two dependent variables: RT and control rating (Figure 8). RT and control rating were normalized within participants (by subtracting the mean and dividing the result by the standard deviation of each participant) to remove individual differences in visual search and rating. In the model, we drew direct paths from the actual control to control rating and RT and paths from control rating to RT. We pooled averaged values in each condition from all participants for the model. The SEMs were performed using IBM SPSS Amos 22 for the two conditions of control context. The standardized coefficients for all paths are depicted in Figure 8. The most important finding was that, in both the contexts of no control and perfect control, control rating significantly influenced RT $(p=.001$
Figure 8. The SEM of the performance of visual search and its predictors. The paths with significant coefficients are shown as solid lines, and the paths with nonsignificant coefficients are shown as broken lines. The model shows that, in both the contexts when the participants had no control and perfect control over the distractors, the subjective feeling of control over the target (i.e., control rating) was significantly linked to the performance of visual search (i.e., RT), even after taking into consideration the direct path from actual control to RT.

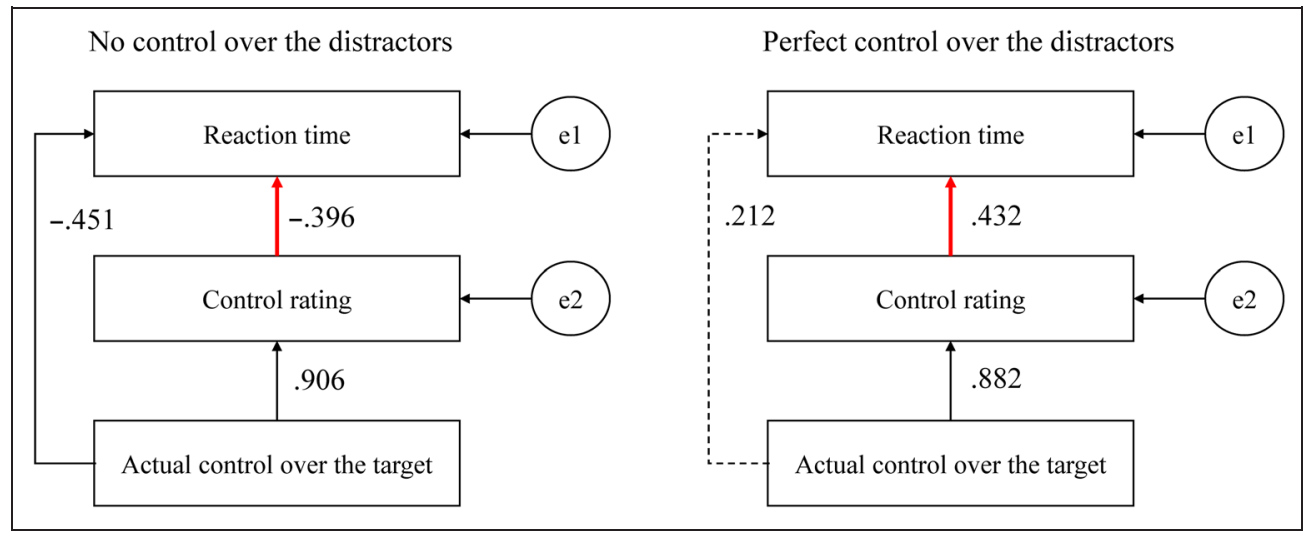


and .004 , respectively), even after taking into consideration the direct path from actual control on RT.

\section{Discussion}

The purpose of Experiment 2 was to examine our hypothesis of high prioritized attention selection on a less controlled object when people have already acquired high level of control, compared with the condition in which people allocate attention to a controllable object among several uncontrollable objects. Our main result supported the hypothesis by showing that attention was drawn to an object that was under slightly less control than other perfectly controlled objects. This case showed significantly lower RTs in visual search than the case where one object was under slightly more control than the other uncontrollable objects. Furthermore, the results of SEM showed that the subjective feeling of control was an important mediator of attentional selection in both conditions when people had or did not have control over the majority of the objects.

In summary, Experiments 1 and 2 provided empirical evidences for our hypotheses of attentional selection on differential control over one object among many and prioritized monitoring of one object with very modestly reduced control when people have acquired a high level of control over many. Moreover, the multivariable analyses in Experiments 1 and 2 further showed that the subjective feeling of control was an important feature for attention allocation. The results of Experiment 1 revealed an important issue that a sudden change in control may capture attention when the change is a decrease rather than when it is an increase in control, which requires further investigation. To examine this issue, we measured the ERPs for the events of losing and gaining control in Experiment 3 to find out whether the neural processes underlying attention allocation for the two types of events is quantitatively or qualitatively different.

\section{EXPERIMENT 3}

\section{Methods}

\section{Participants}

Seventeen healthy volunteers recruited from the same participant database as the previous experiments. All the participants were right-handed and reported normal or corrected-to-normal visual acuity. One participant did not complete the task because of a technical problem and therefore was excluded from the analysis, resulting in a sample size of 16 (mean age $=24.4$, range $=18$ $36, S D=5.9,7$ women). The sample size of 16 was based on a power calculation with an effect size estimated from the results of Experiments 1 and $2(\alpha=.05$, power $(1-$ $\beta$ error $)=.95$ ) using $G^{*}$ Power 3 (Faul et al., 2007). The study was approved by the local ethics committee (University College London). All participants provided written informed consent before participation and received $£ 7.50$ per hour as reimbursement.

\section{Task}

Figure 9 shows the timeline of the experimental trial. Participants pressed the space key to start the trial when they felt ready. Then, ten 2-mm black dots appeared at random positions within $12.5 \mathrm{~mm}$ from the center of a $338 \times 270 \mathrm{~mm}$ (width $\times$ height) gray screen, along with a dark gray cross in the center of the screen. Once participants started to move their right index finger on a touchpad, which was fixed to the desk, all the dots started to move together in the same direction. The velocity, onset, and offset of the dots' movement corresponded to the finger movement, but the spatial relation between finger movement and dot movement could either involve a regular spatial transformation or a replay of prerecorded spatial trajectories, as in Experiments 1 and 2. Participants were instructed to make smooth, continuous, and slow finger movements. They were also told

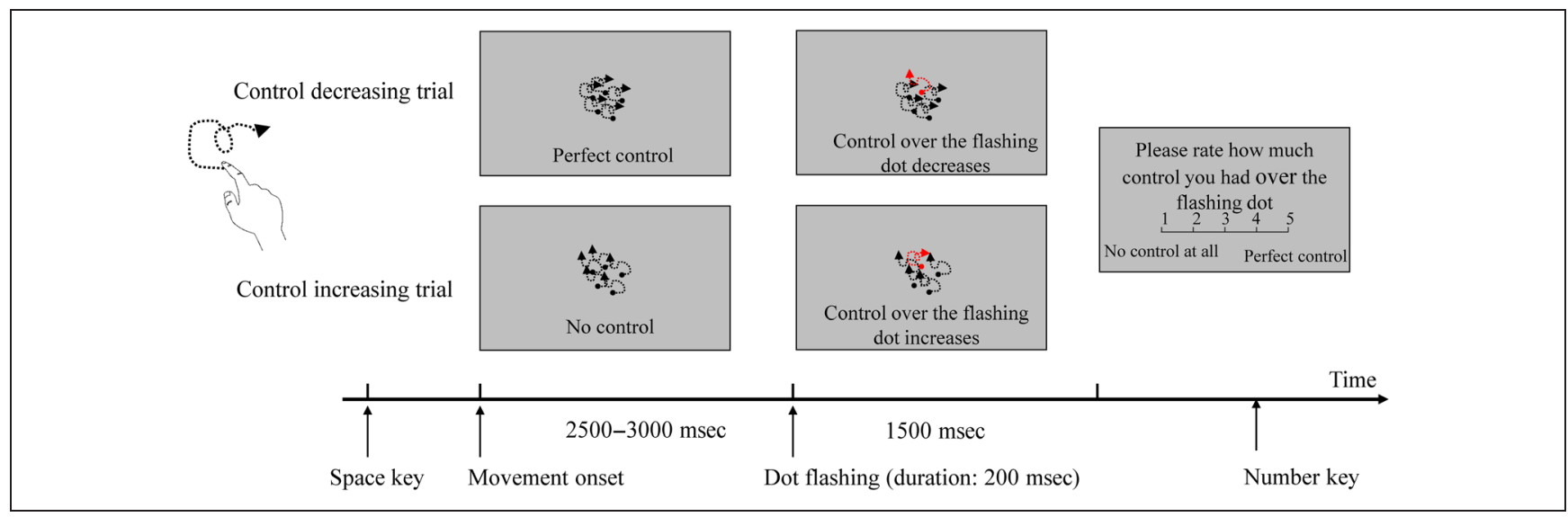

Figure 9. Timeline of the task in Experiment 3. Participants were instructed to move their right index finger continuously and smoothly when they saw the dots. The dots were under $100 \%$ or $0 \%$ control for the first $2.5-3 \mathrm{sec}$, and then one dot shortly flashed to red and the control over it changed by $30 \%, 70 \%$, or $100 \%$. Participants kept on moving their finger to find out how much control they then had over this dot and gave a 5-point rating after the dots disappeared. 
to fix their eyes on the central cross, avoiding eye blinks and eye movements. The direction in which the dots moved was either under $100 \%$ or $0 \%$ control of the finger movement from the start of each trial, as in Experiment 2. After a random duration between 2.5 and $3 \mathrm{sec}$ from the onset of first finger movement, one of the dots briefly flashed red for $200 \mathrm{msec}$, and the control over it changed by $30 \%, 70 \%$, or $100 \%$. Participants were asked to keep on moving their fingers to find out how much control they had over this dot after it had flashed. At 1500 msec later, the dots were replaced by a message on the screen, asking participants to rate how much control they had over the dot after it flashed on a 5-point scale ( $1=$ no control at all; $5=$ perfect control) by pressing a number key on a keyboard. Participants were informed that "perfect control" refers to the condition that the flashing dot moved in the same way as they moved their finger whereas "no control at all" means that the flashing dot moved in a completely different direction from the way they moved their finger.

In summary, we used a 2 (Direction of control changing) $x$ 3 (Magnitude of control changing) within-participant design. All the dots were under either perfect (i.e., 100\%) control or no (i.e., 0\%) control for the first $2.5-3 \mathrm{sec}$. Then the control over one dot changed slightly (30\%), moderately (70\%), or maximally (100\%). EEG signals time-locked to onset of the dot flashing were recorded.

\section{Procedure}

Participants sat $50 \mathrm{~cm}$ from a 17-in. LCD monitor, wore an EEG electrode cap, and placed the left hand on a keyboard and the right hand on a touchpad. After receiving an explanation of the task, participants practiced for 30 trials, containing five trials for each condition, in a random order. The actual task contained 300 trials, including 50 repeats for each condition, and the trial order was randomized between participants. The trials were divided into five blocks, each contains 60, allowing participants to take breaks between blocks. The experiment lasted 110 min on average, including the preparation of the EEG equipment.

\section{EEG Recording and Data Analyses}

EEG was recorded from g.LADYbird active ring electrodes from Fz, FCz, Cz, CPz, Pz, POz, F3, F4, F7, F8, FC1, FC2, FC5, FC6, C3, C4, CP1, CP2, CP5, CP6, P3, P4, O1, and $\mathrm{O} 2$ using g.USBamp amplifiers (g.tec, Austria). Horizontal and vertical eye movements were recorded bipolarly from the outer canthi of both eyes and from above and below the left eye, respectively. EEG signals were digitized at a sample rate of $512 \mathrm{~Hz}$ and referenced online against the right earlobe and were then re-referenced to an average of the left and right mastoids offline. A 0.1-Hz Butterworth high-pass filter was used during the recording.

EEG signals were preprocessed using the EEGLAB toolbox (Delorme \& Makeig, 2004) on MATLAB R2016a (The
MathWorks, Natick, MA). A low-pass filter of $30 \mathrm{~Hz}$ and a notch filter of $50 \mathrm{~Hz}$ were applied. EEG signals were segmented into time-locked epochs ranging from $2000 \mathrm{msec}$ before and $1000 \mathrm{msec}$ after the onset of the flashing. Epochs containing large artifacts $( \pm 250 \mu \mathrm{V})$ was removed, and independent component analysis was used to remove eye movement artifacts. Epochs was then rejected with the automatic epoch rejection of EEGLAB (threshold of $\pm 100 \mu \mathrm{V}$ and maximum $5 \%$ of the total trials to reject per iteration), shortened to 1200 -msec periods, starting $200 \mathrm{msec}$ before and ending $1000 \mathrm{msec}$ after the onset of flashing, and were corrected using a 200-msec prestimulus baseline. At last, EEG signals were averaged into ERPs for each condition. ERP mean amplitudes were computed within 100-200 msec, 190-250 msec, and 250-550 msec time windows for P170 (a P2-like potential), N200, and P300 components, respectively, at midline electrodes $\mathrm{Fz}, \mathrm{Cz}$, and $\mathrm{POz}$.

\section{Results}

\section{Subjective Control Rating}

In Experiment 3, all the stimuli including the flashing dot were presented in central field of view, and the flashing effectively identified the target. Therefore, we did not collect RTs as a measure of attention in this task, because we assume that the participant always noticed the flashing dot rapidly. Figure 10 shows the average control ratings. Comparisons on the rating between the two conditions of control context when the actual control was $30 \%$ and $70 \%$ revealed a significant difference in the $70 \%$ control condition, but no significant difference in the $30 \%$ control condition $(t(15)=5.81, p<.001$, Cohen's $d=0.24 ; t(15)=1.44, p=.171$, Cohen's $d=$ 2.31, respectively). A Bonferroni correction with $p$ of .025 was used for the two conditions compared (this comparison was not possible in all four conditions, because

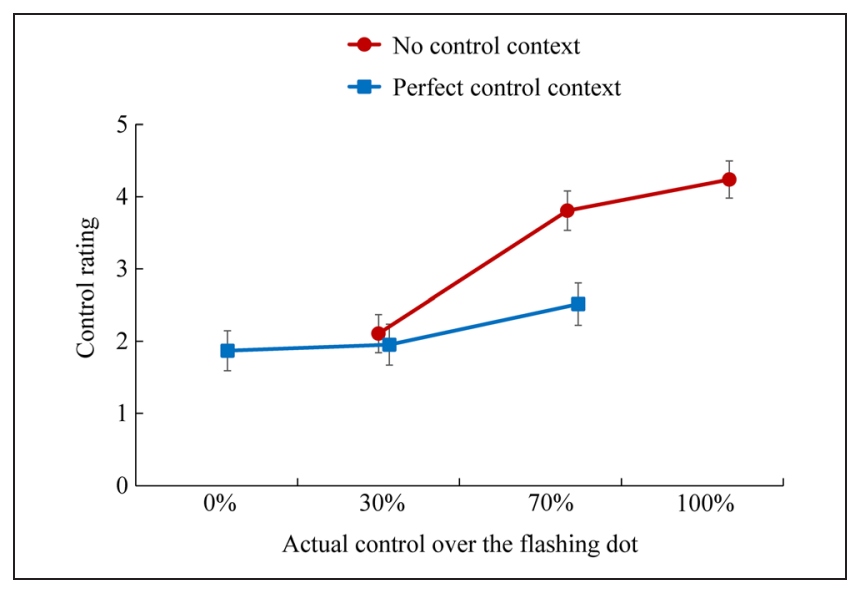

Figure 10. Mean control rating on the flashing dot in Experiment 3. Error bars represent standard errors. The results showed that people felt disproportionate loss of control when actual control decreased from a high level. 
control could not decrease further in the $0 \%$ condition nor increase further in the $100 \%$ condition). The results replicated our findings in Experiment 2 that a small decrement in control greatly diminished the sense of control, comparing to the case when participants gain an equivalent level of control.

\section{ERPs to Control Changing}

Figure 11 shows the ERPs elicited in response to the change in control at midline electrodes $\mathrm{Fz}, \mathrm{Cz}$, and $\mathrm{POz}$, and Figure 12 shows the scalp topographies at the peak latencies of P170, N200, and P300 components. We applied 3 (Electrode, Fz, Cz, or POz) $\times 2$ (Direction of control change, decrease or increase) $\times 3$ (Magnitude of control change, small, large, or maximum) repeatedmeasures ANOVAs to the peak amplitude of each component.

Regarding P170 component, the main effects of Electrode and Direction of change (decrement vs. increment) was significant $\left(F(2,30)=9.79, p=.001\right.$, partial $\eta^{2}=$ $.40 ; F(1,15)=8.55, p=.010$, partial $\eta^{2}=.36$, respectively), whereas the main effect of Magnitude of change, $F(2,30)=1.65, p=.208$, partial $\eta^{2}=.10$, the interaction between Electrode and Direction of change, $F(2,30)=$ $0.45, p>.250$, partial $\eta^{2}=.03$, the interaction between Electrode and Magnitude of change, $F(4,60)=0.61, p>$ .250 , partial $\eta^{2}=.04$, the interaction between Direction and Magnitude of change, $F(2,30)=0.29, p>.250$, partial $\eta^{2}=.02$, and the three-way interaction, $F(4,60)=$ $0.06, p>.250$, partial $\eta^{2}=.004$, were nonsignificant. Post hoc comparisons (Bonferroni-adjusted tests) on electrode showed that the amplitude of P170 at POz was significantly smaller than those at $\mathrm{Fz}$ and $\mathrm{Cz}$ (Bonferroniadjusted $p=.029$ and .004 , respectively). The main effect of Direction of change showed that loss of control elicited significantly larger P170 than gain of control.

Regarding the N200 component, the interaction between Electrode and Magnitude of change was significant, but none of the other main effects or interactions was significant (the main effect of Electrode: $F(2,30)=$ $0.13, p>.250$, partial $\eta^{2}=.01$; the main effect of Direction of change: $F(1,15)=0.13, p>.250$, partial $\eta^{2}=.01$; the main effect of Magnitude of change: $F(2,30)=1.70$, $p=.199$, partial $\eta^{2}=.10$; the interaction of Electrode and Direction of change: $F(2,30)=2.02, p=.151$, partial $\eta^{2}=.12$; the interaction of Electrode and Magnitude of change: $F(4,60)=3.13, p=.021$, partial $\eta^{2}=.17$; the interaction of Direction and Magnitude of change: $F(2$, $30)=0.66, p>.250$, partial $\eta^{2}=.04$; the three-way interaction: $F(4,60)=1.02, p>.250$, partial $\left.\eta^{2}=.06\right)$. Post hoc analysis (Bonferroni-adjusted tests) on the interaction between electrode and magnitude of change revealed larger amplitude of N200 in the condition of maximum change in control than the condition of small change in control at $\mathrm{Fz}$ and $\mathrm{Cz}$, but not at $\mathrm{POz}$.
Finally, regarding the P300 component, the main effects of Electrode, Direction of change, and Magnitude of change were significant $(F(2,30)=6.57, p=.004$, partial $\eta^{2}=.31 ; F(1,15)=8.40, p=.011$, partial $\eta^{2}=.36$; $F(2,30)=11.94, p<.001$, partial $\eta^{2}=.44$, respectively), but none of the interactions between the factors was significant (the interaction between Electrode and Direction of change: $F(2,30)=0.81, p>.250$, partial $\eta^{2}=.05$; the interaction between Electrode and Magnitude of change: $F(4,60)=1.02, p>.250$, partial $\eta^{2}=.06$; the interaction between Direction and Magnitude of change: $F(2,30)=$ $1.19, p>.250$, partial $\eta^{2}=.07$; the three-way interaction: $F(4,60)=0.45, p>.250$, partial $\left.\eta^{2}=.03\right)$. The frontal electrode $\mathrm{Fz}$ showed smaller amplitude than those of more occipital electrodes $\mathrm{Cz}$ and $\mathrm{POz}$ (Bonferroni-adjusted $p=.011$ and .020 , respectively). Moreover, amplitude of P300 was larger in the control decreasing trials than that in the control increasing trials and was larger in the trials with large and maximum changes in control than those with small changes in control (Bonferroni-adjusted $p=$ .003 and .004 , respectively).

In addition, EOGs in Figure 11 showed that participants moved their eyes after the control over the flashing dot changed. The rapid eye movements occurred between 100 and $300 \mathrm{msec}$ after the onset of the change. We conducted a 2 (Direction of control change, decrease or increase) $\times 3$ (Magnitude of control change, small, large, or maximum) repeated-measures ANOVA on the peak of horizontal and vertical EOGs within this time window and did not find any significant main effect or interaction (for vertical EOG, main effect of Direction of change: $F(1,15)=2.79, p=.116$, partial $\eta^{2}=.16$; main effect of Magnitude of change: $F(2,30)=1.44, p>.250$, partial $\eta^{2}=.09$; interaction between Direction and Magnitude of change: $F(2,30)=0.57, p>.250$, partial $\eta^{2}=$ .04; for horizontal EOG, main effect of Direction of change: $F(1,15)=0.68, p>.250$, partial $\eta^{2}=.04 ;$ main effect of Magnitude of change: $F(2,30)=0.03, p>.250$, partial $\eta^{2}<.01$; interaction between Direction and Magnitude of change: $F(2,30)=0.72, p>.250$, partial $\eta^{2}=$ $.05)$. Therefore, the eye movement triggered by the change in stimuli did not differ significantly between conditions.

Furthermore, we conducted a multiple linear regression to predict control rating on the amplitudes of P170 and P300 as independent variables. Because N200 amplitude did not encode information of direction or magnitude of control change, it was omitted from the regression analysis. The averaged response of each condition from each participant was pooled. Regarding the control increasing condition, a significant regression equation was found, $F(2,45)=3.80, p=.030$, with an $R^{2}$ of .145 . The amplitude of $\mathrm{P} 300$ was a significant predictor $(\beta=.343, t(45)=$ $2.48, p=.017)$, but the amplitude of P170 was not $(\beta=$ $.135, t(45)=0.97, p>.250)$. Regarding the control decreasing condition, the regression equation was also significant, $F(2,45)=3.26, p=.047$, with an $R^{2}$ of .127 . 


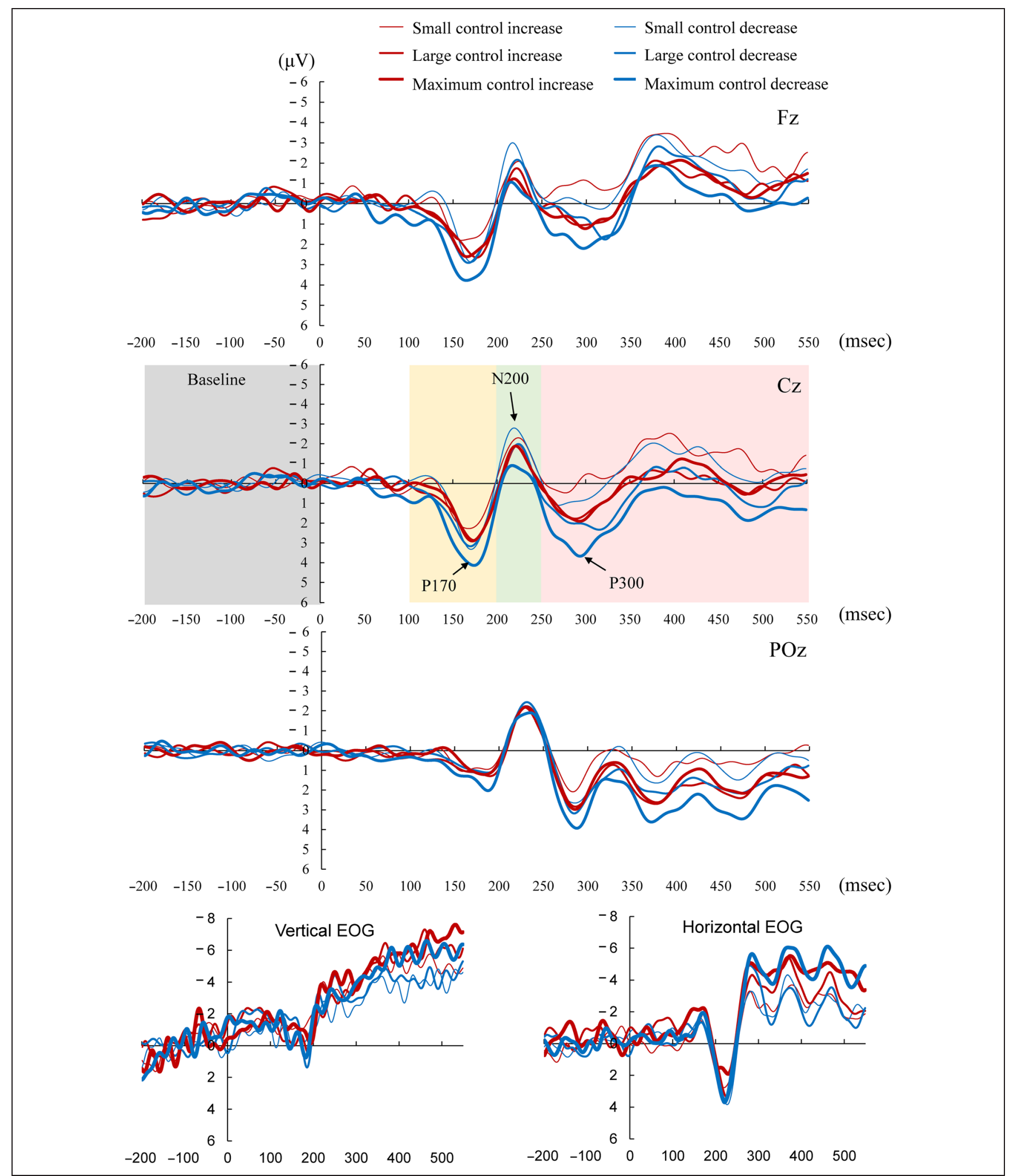

Figure 11. Grand-averaged ERPs in each condition at midline electrodes $\mathrm{Fz}, \mathrm{Cz}$, and POz and averaged vertical and horizontal EOGs elicited in response to the onset of dot flashing and control change. The $x$ axes represent the time relative to flashing onset, the $y$ axes plot activation in $\mu \mathrm{V}$. The gray square represents time window for baseline correction. The yellow, green, and pink areas represent the time windows for P170, N200, and $\mathrm{P} 300$, respectively. 


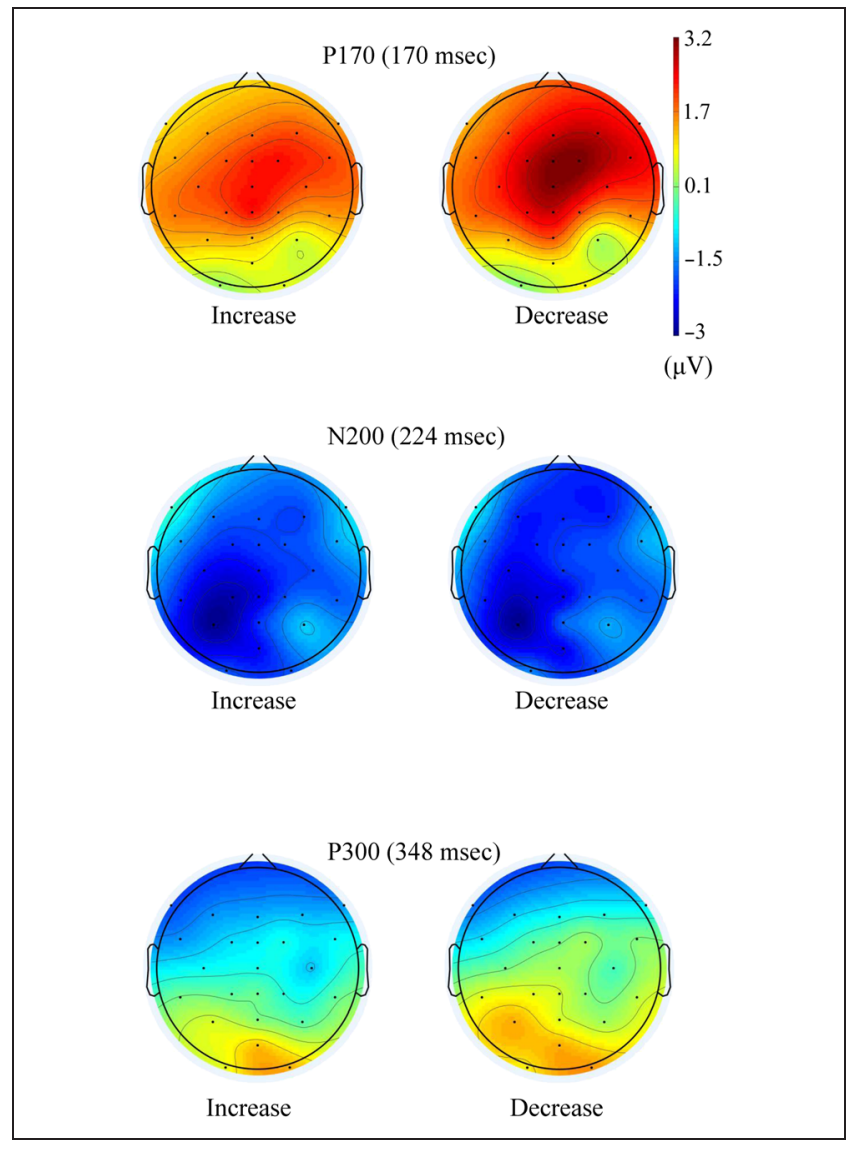

Figure 12. Scalp distribution of P170, N200, and P300 for the control decreasing and increasing conditions.

P300 was a significant predictor $(\beta=-0.309, t(45)=$ $-2.17, p=.035)$, but P170 was not $(\beta=.246, t(45)=$ $1.74, p=.089)$. The results of the multiple linear regression showed that P300 component was linked to the subjective feeling of control, consistent with previous studies (Bednark \& Franz, 2014; Kühn et al., 2011).

To summarize, results from Experiment 3 showed that a decrease of control elicited larger P170 and P300, compared with an increase of control, and larger changes in control (both increases and decreases) were linked with larger P300 amplitudes, compared with small changes in control.

\section{Discussion}

The purpose of Experiment 3 was to quantify attentional processing of an increase or decrease of control. ERPs provide useful indices for examining both the quantitative (i.e., the extent of attention) and qualitative (i.e., the phase of attention) aspects of attention. We found that the event of losing control of an already controlled object elicited larger P170 and P300 than the event of gaining an equivalent amount of control over an uncontrolled object. We conclude that monitoring for loss of control takes more priority in early attentional processing than monitoring for gain of control. Furthermore, control changes with larger magnitude elicited larger P300 compared with small changes, supporting our hypothesis that the monitoring of control produces triggers to attention regarding the extent of difference in control.

P170 is a frontal potential that can be identified with previous reports of a P200 or P2. Previous studies reported that $\mathrm{P} 2$ peak amplitude was larger in response to feedback perturbation in auditory stimulus during active vocalization than passive listening (Behroozmand \& Larson, 2011; Behroozmand, Karvelis, Liu, \& Larson, 2009), indicating that P2 might reflect the error monitoring under the framework of feed-forward model (Blakemore et al., 1998). Furthermore, a recent study found that P2 was enhanced when the self-generated sound was delayed for $\leq 200$ msec, but the enhancement of P2 diminished in long-delay conditions (Toida, Ueno, \& Shimada, 2016), indicating that P2 might reflect the control-error monitoring (when there is still some degree of control) rather than the deviance in outcomes. Our results of finding larger P170 in the control decreasing trials than the control increasing trials support the assertion that P170 may be associated with error monitoring related to the self (Adler, Schabinger, Michal, Beutel, \& Gillmeister, 2016).

On the other hand, P300 is a widely studied potential, which has been attributed to attention allocation processes (Polich, 2007; Picton, 1992). For example, larger amplitude of P300 was linked to attention to self-referential stimuli (e.g., Tacikowski \& Nowicka, 2010; Gray, Ambady, Lowenthal, \& Deldin, 2004). In the present experiment, we used P300 as an index to examine attention to objects that differed in direction and magnitude of a change in control. The results clearly showed that loss of control from a perfectly controlled context lead to enhanced neural processing compared with a gain of control from a context of no control. The larger these changes were, the greater the effect on neural processing. In summary, the results showed that the gains and losses of control are not only processed in both quantitatively and also qualitatively different ways in the allocation of attention.

\section{GENERAL DISCUSSION}

We have studied how controlling an external object through one's own actions influences visual attention to that object. We hypothesized that people pay attention to an object under more control than other objects when they do not have much control over the external world. On the other hand, once people acquire a high level of control over the external world, any loss of control becomes very salient. The hypothesis was well supported by the results from the three experiments. In Experiment 1, most objects were under partial, $50 \%$, control. In these conditions, an object under more control was noticeable whereas an object under less control was not. Furthermore, Experiment 2 found that when people already had perfect control over the external world, a slight lack of control 
rapidly captured attention. Finally, a decrease of control elicited larger P170 and P300 than equivalent increases of control, indicating a high priority for loss of control in attentional processing.

Attention has been known to widely influence most of our cognitive processes, including the sense of control (Wen, Yamashita, \& Asama, 2016; Hon, Poh, \& Soon, 2013). However, the opposite question, how the sense of control shapes attention and, through it, affects wider cognitive processing, has been less studied. Our results suggested that the sense of control over external objects strongly influences how people perceive those objects. First, when actual control is minimal, a more controlled object attracts attention. This suggests that people pay attention to objects that they may potentially be able to control. Search for control may reflect a cognitive primitive, similar to the search for information (Hikosawa, Miyauchi, \& Shimojo, 1993). On the other hand, once control is registered, perceptual processing is highly sensitive to even very small losses of control. This pattern of results is consistent with the notion that control over external objects is a form of internal reward, which is carefully conserved by specific cognitive and behavioral processes. This view was initially supported by preference and increased vigour for actions associated that involve control of an external object, compared with actions that do not (Karsh \& Eitam, 2015; Eitam, Kennedy, \& Tory Higgins, 2013). Our results were obtained in tasks without overt preference or reward. Nevertheless, our finding of precise monitoring to discover and then maintain sense of control over an external object is consistent with this view. In interacting with the external world, we want to have control and do not then want to lose it.

Previous behavioral and neural studies have used comparator models of motor control (Blakemore et al., 1998, 1999, 2002; Wolpert \& Flanagan, 2001; Frith et al., 2000; Wolpert \& Ghahramani, 2000) to investigate the causes of the "sense of agency." These studies generally agree that sense of agency results from strong spatial and/or temporal match between the intended consequence of an action (in this case, a finger movement) and the actual consequences. However, these studies have rarely considered the wider consequences of having agency beyond explicit (Daprati et al., 1997) or implicit (Haggard, Clark, \& Kalogeras, 2002) measures of agency itself. One possible exception comes from studies of "sensory attenuation" effects of agency. This reduction in perceptual intensity of self-generated events (von Holst \& Mittelstaedt, 1950) is often explained by the intuition that a sensory event that can be accurately predicted is not a priority for perception. In contrast, an unexpected event or "prediction error" would signal a lack of agency and is a priority for perception. Such nonagency events were found to activate an agency-monitoring network focused on the angular gyrus in the parietal cortex (Sperduti, Delaveau, Fossati, \& Nadel, 2011; Farrer et al., 2008; Farrer \& Frith, 2002).
Here we have considered how agency affects attention. Our finding of attentional facilitation for reductions in control is broadly consistent with the prediction error account and the comparator model. When a visual object is already under control, a reduction in the degree of control will trigger a prediction error. One consequence of the prediction error might be a relative boost in the perceived intensity and/or salience of the visual object, which would in turn attract attention. Because we have not measured perceived intensity directly, the link between intensity and attentional salience remains a plausible hypothesis, rather than an established fact. However, we also found that gaining control over an object in the context of lack of control also facilitated attention. This latter effect, in contrast, seems contrary to the predictions of the comparator model. Actually, the results from Experiment 2 showed that attention was most attracted to the target when it was under perfect control and the distractors were under no control, compared with the condition when the target was under no control and the distractors were under perfect control (Figure 6). Thus, the effects of control on attention do not simply follow the sensory attenuation principle. We suggest that they rather reflect an attentional selection for objects on the basis of their distinctive visual motion properties. Monitoring of visual motion may be facilitated when visual motion is closely spatially related to one's own hand movement, thus explaining the attentional facilitation from both gaining control in the context of no control and losing control in the context of overall control. In addition, participants were asked to rate the level of control in all three experiments. This presumably drew attention to the control over stimuli. Future studies might usefully examine whether control over an object draws attention to it automatically, even in the absence of any task requirement to make judgments of control.

Lastly, in daily life, the detection of control may be much easier than that in our tasks, as one could use both temporal and spatial features of movements. For instance, to recognize oneself in a live video of a crowd, a useful strategy is to wave one's hands. One then pops out, as long as nobody else does the same action at the same time. Laboratory studies confirm that synchrony of action onset and visual motion onset is an extremely potent cue for agency (Tsakiris, Longo, \& Haggard, 2010). However, in our task, we created a situation in which temporal information about motion onsets and offsets is not sufficient for detecting control. To pursue our illustrative example, if one waved one's hands, all the people in the video would wave simultaneously with the same speed. To recognize oneself in such case, one must compare the spatial pattern of one's own pattern with the spatial pattern of the visual input. We failed to find the dramatic self pop-out effects seen in some agency tasks, perhaps because spatial patterns of motion are a weaker cue for control than temporal patterns of motion onsets and offsets. However, our results showed that, with only spatial cues, 
people can still detect and monitor control and allocate attention to it efficiently.

\section{Acknowledgments}

The authors thank Dr. Frederike Beyer for her fruitful comments on the manuscript. This study was supported by ERC Advanced Grant HUMVOL. W. W. was supported by the Uehara Memorial Foundation and Marubun Research Promotion Foundation.

Reprint requests should be sent to Wen Wen, Institute of Cognitive Neuroscience, University College London, Alexandra House, 17 Queen Square, London WC1N 3AZ, UK, or via e-mail:w.wen@ucl.ac.uk.

\section{Notes}

1. Please see https://youtu.be/uP3gjFEYDBU for a demonstration video of the task.

2. Please see https://youtu.be/MVeKbz6Ic3c for a demonstration video of Experiment 2.

\section{REFERENCES}

Adler, J., Schabinger, N., Michal, M., Beutel, M. E., \& Gillmeister, H. (2016). Is that me in the mirror? Depersonalisation modulates tactile mirroring mechanisms. Neuropsychologia, 85, 148-158.

Band, G. P. H., van Steenbergen, H., Ridderinkhof, K. R., Falkenstein, M., \& Hommel, B. (2009). Action-effect negativity: Irrelevant action effects are monitored like relevant feedback. Biological Psychology, 82, 211-218.

Bednark, J. G., \& Franz, E. A. (2014). Agency attribution: Eventrelated potentials and outcome monitoring. Experimental Brain Research, 232, 1117-1126.

Behroozmand, R., Karvelis, L., Liu, H., \& Larson, C. R. (2009) Vocalization-induced enhancement of the auditory cortex responsiveness during voice F0 feedback perturbation. Clinical Neurophysiology, 120, 1303-1312.

Behroozmand, R., \& Larson, C. R. (2011). Error-dependent modulation of speech-induced auditory suppression for pitch-shifted voice feedback. BMC Neuroscience, 12, 54.

Blakemore, S.-J., Frith, C. D., \& Wolpert, D. M. (1999). Spatio-temporal prediction modulates the perception of self-produced stimuli. Journal of Cognitive Neuroscience, 11, 551-559.

Blakemore, S.-J., Wolpert, D. M., \& Frith, C. D. (1998). Central cancellation of self-produced tickle sensation. Nature Neuroscience, 1, 635-640.

Blakemore, S.-J., Wolpert, D. M., \& Frith, C. D. (2002). Abnormalities in the awareness of action. Trends in Cognitive Sciences, 6, 237-242.

Botvinick, M. M., Cohen, J. D., \& Carter, C. S. (2004). Conflict monitoring and anterior cingulate cortex: An update. Trends in Cognitive Sciences, 8, 539-546.

Botvinick, M. M., Niv, Y., \& Barto, A. C. (2009). Hierarchically organized behavior and its neural foundations: A reinforcement-learning perspective. Cognition, 113, 262-280.

Chambon, V., Wenke, D., Fleming, S. M., Prinz, W., \& Haggard, P. (2013). An online neural substrate for sense of agency. Cerebral Cortex, 23, 1031-1037.

Daprati, E., Franck, N., Georgieff, N., Proust, J., Pacherie, E., Dalery, J., et al. (1997). Looking for the agent: An investigation into consciousness of action and self-consciousness in schizophrenic patients. Cognition, 65, 71-86.

Delorme, A., \& Makeig, S. (2004). EEGLAB: An open sorce toolbox for analysis of single-trail EEG dynamics including independent component analysis. Journal of Neuroscience Methods, 134, 9-21.

Eitam, B., Kennedy, P. M., \& Tory Higgins, E. (2013). Motivation from control. Experimental Brain Research, 229, 475-484.

Farrer, C., Franck, N., Georgieff, N., Frith, C. D., Decety, J., \& Jeannerod, M. (2003). Modulating the experience of agency: A positron emission tomography study. Neuroimage, 18, 324-333.

Farrer, C., Frey, S. H., Van Horn, J. D., Tunik, E., Turk, D., Inati, S., et al. (2008). The angular gyrus computes action awareness representations. Cerebral Cortex, 18, 254-261.

Farrer, C., \& Frith, C. D. (2002). Experiencing oneself vs. another person as being the cause of an action: The neural correlates of the experience of agency. Neuroimage, 15, 596-603.

Faul, F., Erdfelder, E., Lang, A.-G., \& Buchner, A. (2007). $G^{*}$ Power 3: A flexible statistical power analysis program for the social, behavioral, and biomedical sciences. Behavior Research Methods, 39, 175-191.

Frith, C. D., Blakemore, S.-J., \& Wolpert, D. M. (2000). Explaining the symptoms of schizophrenia: Abnormalities in the awareness of action. Brain Research Reviews, 31, 357-363.

Goodale, M. A. (2014). How (and why) the visual control of action differs from visual perception. Proceedings of the Royal Society, Series B, Biological Sciences, 281, 20140337.

Goodale, M. A., \& Milner, A. D. (2004). Sight unseen: An exploration of conscious and unconscious vision. Oxford: Oxford University Press.

Gray, H. M., Ambady, N., Lowenthal, W. T., \& Deldin, P. (2004). P300 as an index of attention to self-relevant stimuli. Journal of Experimental Social Psychology, 40, 216-224.

Haggard, P. (2017). Sense of agency in the human brain. Nature Reviews Neuroscience, 18, 197-208.

Haggard, P., \& Chambon, V. (2012). Sense of agency. Current Biology, 22, R390-R392.

Haggard, P., Clark, S., \& Kalogeras, J. (2002). Voluntary action and conscious awareness. Nature Neuroscience, 5, 382-385.

Hikosawa, O., Miyauchi, S., \& Shimojo, S. (1993). Voluntary and stimulus induced atention detected as motion sensation. Perception, 22, 517-526.

Hon, N., Poh, J.-H., \& Soon, C.-S. (2013). Preoccupied minds feel less control: Sense of agency is modulated by cognitive load. Consciousness and Cognition, 22, 556-561.

Karsh, N., \& Eitam, B. (2015). I control therefore I do: Judgments of agency influence action selection. Cognition, 138, 122-131.

Keenan, J. P., McCutcheon, B., Freund, S., Gallup, G. G., Sanders, G., \& Pascual-Leone, A. (1999). Left hand advantage in a self-face recognition task. Neuropsychologia, 37, 1421-1425.

Kühn, S., Brass, M., \& Haggard, P. (2013). Feeling in control: Neural correlates of experience of agency. Cortex, 49 , 1935-1942.

Kühn, S., Nenchev, I., Haggard, P., Brass, M., Gallinat, J., \& Voss, M. (2011). Whodunnit? Electrophysiological correlates of agency judgements. PLOS ONE, 6, e28657.

Kumar, D., \& Srinivasan, N. (2014). Naturalizing sense of agency with a hierarchical event-control approach. PLOS ONE, 9, e92431.

Kumar, D., \& Srinivasan, N. (2017). Multi-scale control influences sense of agency: Investigating intentional binding using event-control approach. Consciousness and Cognition, 49, $1-14$.

Kumar, N., Manjaly, J. A., \& Sunny, M. M. (2015). The relationship between action-effect monitoring and attention capture. Journal of Experimental Psychology: General, 144, 18-23.

Metcalfe, J., Eich, T. S., \& Miele, D. B. (2013). Metacognition of agency: Proximal action and distal outcome. Experimental Brain Research, 229, 485-496. 
Metcalfe, J., \& Greene, M. J. (2007). Metacognition of agency. Journal of Experimental Psychology: General, 136, 184-199.

Ogawa, K., \& Inui, T. (2007). Lateralization of the posterior parietal cortex for internal monitoring of self- versus externally generated movements. Journal of Cognitive Neuroscience, 19, 1827-1835.

O'Regan, J. K. (1992). Solving the "real" mysteries of visual perception: The world as an outside memory. Canadian Journal of Psychology, 46, 461-488.

Picton, T. W. (1992). The P300 wave of the human eventrelated potential. Journal of Clinical Neurophysiology, 9, 456-479.

Polich, J. (2007). Updating P300: An integrative theory of P3a and P3b. Clinical Neurophysiology, 118, 2128-2148.

Raudenbush, S. W., Bryk, A. S., Cheong, Y. F., Congdon, R., \& Du Toit, M. (2011). Hierarchical linear and nonlinear modeling (HLM7). Lincolnwood, IL: Scientific Software International.

Rogers, T. B., Kuiper, N. A., \& Kirker, W. S. (1977). Selfreference and the encoding of personal information. Journal of Personality and Social Psychology, 35, 677-688.

Salomon, R., Lim, M., Kannape, O., Llobera, J., \& Blanke, O. (2013). "Self pop-out": Agency enhances self-recognition in visual search. Experimental Brain Research, 228, 173-181.

Salomon, R., Szpiro-Grinberg, S., \& Lamy, D. (2011). Selfmotion holds a special status in visual processing. PLOS ONE, 6, 2-8.

Schneider, W., \& Shiffrin, R. M. (1977). Controlled and automatic human information processing: I. Detection, search, and attention. Psychological Review, 84, 1-66.

Sperduti, M., Delaveau, P., Fossati, P., \& Nadel, J. (2011). Different brain structures related to self- and external-agency attribution: A brief review and meta-analysis. Brain Structure E Function, 216, 151-157.
Tacikowski, P., \& Nowicka, A. (2010). Allocation of attention to self-name and self-face: An ERP study. Biological Psychology, 84, 318-324.

Toida, K., Ueno, K., \& Shimada, S. (2016). Neural basis of the time window for subjective motor-auditory integration. Frontiers in Human Neuroscience, 9, 1-12.

Tsakiris, M., Longo, M. R., \& Haggard, P. (2010). Having a body versus moving your body: Neural signatures of agency and body-ownership. Neuropsychologia, 48, 2740-2749.

von Holst, E., \& Mittelstaedt, H. (1950). Das Reaffernzprinzip wechselwirkungen zwichen zentrainervensystem und peripherie. Naturwissenschalten, 37, 464-476.

Wegner, D. M. (2002). The illusion of conscious will. Cambridge MA: MIT Press.

Wen, W., Yamashita, A., \& Asama, H. (2015a). The influence of goals on sense of control. Consciousness and Cognition, 37, 83-90.

Wen, W., Yamashita, A., \& Asama, H. (2015b). The sense of agency during continuous action: Performance is more important than action-feedback association. PLOS ONE, 10, e0125226.

Wen, W., Yamashita, A., \& Asama, H. (2016). Divided attention and processes underlying sense of agency. Frontiers in Psychology, 7, 35.

Wen, W., Yamashita, A., \& Asama, H. (2017). The influence of performance on action-effect integration in sense of agency. Consciousness and Cognition, 53, 89-98.

Whiteley, L., Spence, C., \& Haggard, P. (2008). Visual processing and the bodily self. Acta Psychologica, 127, 129-136.

Wolpert, D. M., \& Flanagan, J. R. (2001). Motor prediction. Current Biology, 11, R729-R732.

Wolpert, D. M., \& Ghahramani, Z. (2000). Computational principles of movement neuroscience. Nature Neuroscience, 3(Suppl.), 1212-1217. 